\section{Necrotising pneumonia and empyema due to Clostridium perfringens complicating pulmonary embolus}

\author{
Y Bashir, M K Benson
}

\begin{abstract}
A fatal case of necrotising pneumonia due to Clostridium perfringens developing as a complication of pulmonary infarction is reported.
\end{abstract}

Clostridial pneumonia classically develops as a complication of penetrating chest injuries. ${ }^{1}$ Primary clostridial pleuropulmonary infection is uncommon but recognised ${ }^{2}$ and has usually been attributed to either aspiration or contamination of the pleural cavity by percutaneous procedures. We report a patient in whom the likely mechanism was secondary infection of a pulmonary infarct.

\section{Case report}

A previously fit 66 year old man was admitted with a three week history of right sided pleuritic chest pain, progressive dyspnoea, and recurrent haemoptysis. He smoked 30 cigarettes a day and had no predisposing factors for venous thromboembolism.

On examination he appeared unwell but was afebrile. His pulse rate was $110 / \mathrm{min}$ and blood pressure $100 / 75 \mathrm{~mm} \mathrm{Hg}$. There was dullness to percussion and bronchial breathing over the Dr Y Bashir, Department of Cardiological Sciences, $\mathrm{St}$ George's Hospital Medical School, London SW17 ORE Accepted 5 October 1989

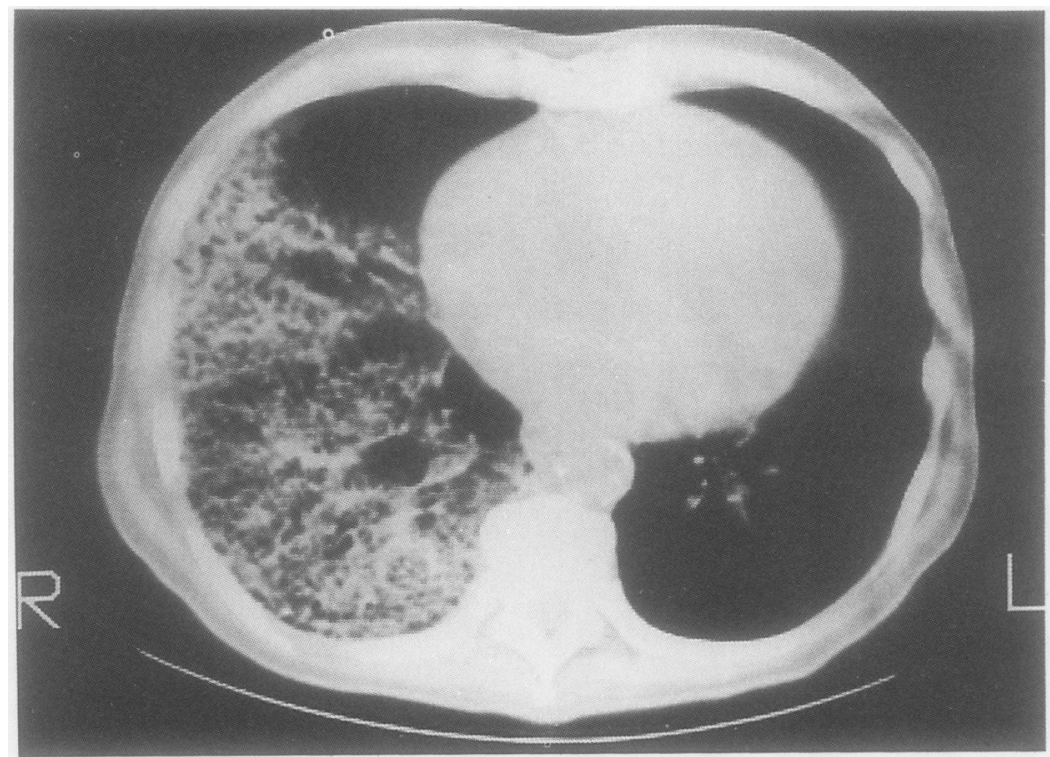

Lower thoracic computed tomogram five days after admission showing extensive consolidation and cavitation in the right lower lobe. right lung base. Physical examination otherwise showed nothing abnormal.

A chest radiograph at admission showed consolidation in the right lower lobe with a small effusion. Arterial blood gases while he was breathing air were: $\mathrm{pH} 7 \cdot 48$, carbon dioxide tension $4 \cdot 1 \mathrm{kPa}$, oxygen tension $10.5 \mathrm{kPa}$. Bronchial carcinoma was suspected, but no lesion was seen at bronchoscopy and cytological examination of sputum and pleural fluid gave negative results. A ventilation-perfusion scan showed bilateral mismatched perfusion defects and was highly suggestive of pulmonary emboli. Subsequently the pleural aspirate yielded a pure growth of Clostridium perfringens.

The presumptive diagnosis was secondary infection of a pulmonary infarct by $C$ perfringens and he was treated with intravenous benzylpenicillin and metronidazole. Heparinisation was deferred because of recurrent haemoptysis. Forty eight hours later his condition had not improved, and by this time there was extensive consolidation and cavitation of the right lower lobe with a subpulmonary collection of gas and fluid (figure). The pleural cavity was drained via an intercostal tube but he continued to deteriorate and became jaundiced, hypotensive, and oliguric. On the 12th day after admission he was transferred to the intensive care unit and died soon after arrival. Permission for a postmortem examination was refused

\section{Discussion}

Secondary infection of a pulmonary infarct is a serious complication that may result in abscess formation or empyema and often requires surgical drainage. ${ }^{3}$ On the basis of postmortem series the incidence of superinfection in pulmonary infarcts has been estimated at $2 \cdot 7 \%$, though some of these cases may have been caused by septic emboli in the first place. ${ }^{4}$

Clostridia may be isolated from a chronically infected lung or pleural space in a mixed bacterial culture, ${ }^{56}$ but here it is doubtful whether they have caused the infection. Primary clostridial pleuropulmonary infection usually takes the form of a necrotising pneumonia with abscess formation and empysema. ${ }^{278}$ It has been suggested that the organism is introduced by aspiration or during percutaneous procedures affecting the pleura, but in some patients there is no evidence for either mechanism. In the present case there was probably haematogenous infection of a pulmonary infarct; necrotic lung tissue would constitute an ideal environment for anaerobic or microaerophilic growth. It seems less likely that the pulmonary emboli and clostridial pneumonia were unrelated.

A recent review listed 18 reported cases of clostridial pleuropulmonary infection ${ }^{2}$ and in two of these patients pulmonary emboli were documented by lung scintigraphy or necropsy. ${ }^{89}$ In two other cases pulmonary thromboembolic disease was excluded by angiography. ${ }^{710}$ In the remaining cases it was not mentioned as a possibility even though 
pleuritic chest pain, dyspnoea, and haemoptysis were common presenting symptoms.

All three reported cases of clostridial pneumonia in association with pulmonary emboli (refs 8 and 9 and the present case) have resulted in death, emphasising the severity of this complication. These patients need vigorous treatment with parenteral antibiotics and early chest drainage to have a chance of survival. Urgent resection of necrotic lung is a further therapeutic option but we are not aware of any previous experience with this approach.

We are grateful to Dr F Wright, Radiology Department, Churchill Hospital, Oxford, for permission to reproduce the computed tomogram.
1 Elliott TR, Henry $\mathrm{H}$. Infection of haemothorax by anaerobic gas-producing bacilli. $\mathrm{Br}$ Med $J$ 1917;1:413-8.

Bekemeyer WB. Clostridial infections of the lungs and pleura. Southern Med J 1986;79:1393-7.

3 May IA, Samson PC, Mittal A. Surgical management of the patient with complications of pulmonary infarction due to nonseptic pulmonary emboli. Am J Surg 1972;124:223-7.

4 Spencer H. Pathology of the lung. Oxford: Pergamon Press, 1985:629-70.

5 Finland M, Barnes MW. Changing ecology of acute bacterial empyema: occurrence and mortality at Boston City Hospital during 12 selected years from 1935 to 1972 . Infect Dis 1978;137:274-91.

6 Bartlett JC, Finegold SM. Anaerobic infections of the lung and pleural space. Am Rev Respir Dis 1974;110:56-77.

7 Bayer AS, Nelson SC, Galpin JE, et al. Necrotising pneumonia and empyema due to Clostridium perfringens. pneumonia and empyema

8 Spagnulo PJ, Payne VD. Clostridial pleuropulmonary infection. Chest 1980;78:622-5

9 Misra DP, Hurst DJ. Necrotising pneumonia and empyema caused by Clostridium bifermentans. Thorax 1980;35 $310-1$.

10 Malmorg A, Rylander M, Selander H. Primary thoracic empyema caused by Clostridium sporogenes. Scand J Infect Dis 1970;2:155-6. 